\title{
Complexity of the Multi-Service Center Problem*
}

\author{
${\text { Takehiro } \text { Ito }^{1} \text {, Naonori Kakimura }}^{2}$, and Yusuke Kobayashi ${ }^{3}$ \\ 1 Graduate School of Information Sciences, Tohoku University, Sendai, Japan \\ takehiro@ecei.tohoku.ac.jp \\ 2 Department of Mathematics, Keio University, Yokohama, Japan \\ kakimura@math.keio.ac.jp \\ 3 Faculty of Engineering, Information and Systems, University of Tsukuba, \\ Tsukuba, Japan. \\ kobayashi@sk.tsukuba.ac.jp
}

\begin{abstract}
The multi-service center problem is a variant of facility location problems. In the problem, we consider locating $p$ facilities on a graph, each of which provides distinct service required by all vertices. Each vertex incurs the cost determined by the sum of the weighted distances to the $p$ facilities. The aim of the problem is to minimize the maximum cost among all vertices. This problem is known to be NP-hard for general graphs, while it is solvable in polynomial time when $p$ is a fixed constant. In this paper, we give sharp analyses for the complexity of the problem from the viewpoint of graph classes and weights on vertices. We first propose a polynomial-time algorithm for trees when $p$ is a part of input. In contrast, we prove that the problem becomes strongly NP-hard even for cycles. We also show that when vertices are allowed to have negative weights, the problem becomes NP-hard for paths of only three vertices and strongly NP-hard for stars.
\end{abstract}

1998 ACM Subject Classification G.2.2 Graph Theory

Keywords and phrases facility location, graph algorithm, multi-service location

Digital Object Identifier 10.4230/LIPIcs.ISAAC.2017.48

\section{Introduction}

Facility location is one of the most well-studied topics in combinatorial optimization. There are various kinds of settings depending on the situations. (See e.g., [4].) Generally, in facility location problems, we are given a set of clients and a set of facilities in a graph, and we aim to decide which facilities are open to satisfy the demand of the clients. For example, the well-known $k$-center problem is to place $k$ facilities in a graph so that the maximum distance from each client to their closest facility is minimized $[6,7]$. Note that this standard situation assumes that all $k$ facilities can provide the same service so that each client meets their demand by only accessing one facility.

$\mathrm{Yu}$ and $\mathrm{Li}$ [10] recently proposed a new framework of facility location problems, called multi-service location problems, motivated by the situation where each facility provides different services and each client needs to access all facilities to meet their demand. As the first problem of this kind, they proposed the $p$-SERVICE CENTER problem defined as follows. (The formal definition will be given in Section 2.) In the problem, we assume that clients

\footnotetext{
* This work is partially supported by JST ERATO Grant Number JPMJER1201, JST CREST Grant Number JPMJCR1402, and JSPS KAKENHI Grant Numbers JP16H03118, JP16K00004, JP16K16010 and JP17K00028, Japan.
}

(c) (i) Takehiro Ito, Naonori Kakimura, and Yusuke Kobayashi;

c. licensed under Creative Commons License CC-BY

28th International Symposium on Algorithms and Computation (ISAAC 2017).

Editors: Yoshio Okamoto and Takeshi Tokuyama; Article No. 48; pp. 48:1-48:12

Leibniz International Proceedings in Informatics

LIPICS Schloss Dagstuhl - Leibniz-Zentrum für Informatik, Dagstuhl Publishing, Germany 
are all vertices in a graph $G$, and facilities can be located on any place in $G$ even on an edge. When we locate $p$ facilities, each of which provides distinct service, the cost of each client $v$ is determined by the sum of the weighted distances to the $p$ facilities, where the weighted distance from $v$ to a facility $x$ is the shortest-path distance from $v$ to $x$ multiplied by a positive weight (representing the demand) of $v$ to the service provided by $x$. The aim of the problem is to find a location of $p$ facilities that minimizes the maximum cost among the clients.

Yu and Li [10] studied the computational complexity of $p$-SERVICE CENTER for several cases. They designed a polynomial-time algorithm for general graphs when $p$ is a fixed constant, and an $O(n \log n)$-time algorithm for trees having $n$ vertices when restricted to $p=2$. On the negative side, they showed that the problem is NP-hard for general graphs when $p$ is a part of input. Anzai et al. [1] showed that this case remains NP-hard even for split graphs with identical edge-length.

In this paper, we consider a simple generalization of $p$-SERVICE CENTER, that is, each client can have zero or negative weights (demands) to a facility; recall that the weight must be positive in the original setting. This generalization is very simple, but enables us to express several natural situations: a zero demand means that the client does not need the service provided by the facility, while a negative demand means that the client refuses the service provided by the facility; furthermore, any vertex can be a non-client by setting all demands to be zero. In this paper, we sharply analyze the computational complexity of this generalized problem from the viewpoint of graph classes and weights of vertices. Our main contributions are summarized as follows:

(1) The problem with nonnegative weights is solvable in polynomial time for trees, even when the number $p$ of facilities is a part of input.

(2) The problem with nonnegative weights is strongly NP-hard for cycles with identical edge-length. Thus, the problem cannot be solved in pseudo-polynomial time even for a cycle unless $\mathrm{P}=\mathrm{NP}$.

(3) When clients are allowed to have negative weights, the problem becomes NP-hard even for paths of only three vertices and strongly NP-hard for stars.

Thus, the problem is polynomially solvable only for trees with nonnegative weights, and is computationally intractable even for a bit larger graph class or negative weights. Let us remark that, while both of the algorithms by Yu and Li [10] require that the number $p$ of facilities is a fixed constant, our algorithm in (1) allows to have $p$ as a part of input.

The rest of the paper is organized as follows. In Section 2, we give a formal definition of the problem studied in this paper. In Section 3, we present a polynomial-time algorithm on a tree. Section 4 is devoted to showing the hardness results.

\section{Problem Definition}

In this section, we formally define the problem studied in this paper.

Let $G=(V, E)$ be an undirected connected graph. For a subgraph $H$ of $G$, we sometimes denote by $V(H)$ and $E(H)$ the vertex set and edge set of $H$, respectively. Assume that each edge $e \in E$ has a length $\ell_{e} \in \mathbb{R}_{\geq 0}$, where $\mathbb{R}_{\geq 0}$ is the set of all nonnegative real numbers. We may assume that all vertices in $G$ are clients, and each facility can be located on any place in $G$, even on an edge. We will refer to interior locations on an edge $e \in E$ by their distances along $e$ from its two endpoints. Throughout the paper, a point on $G$ indicates either a vertex in $V$ or an interior location on an edge in $E$. For notational convenience, we sometimes denote simply by $G$ the set of all points on the graph. For two points $x, y \in G$, let $\operatorname{dist}(x, y)$ denote the shortest-path length between $x$ and $y$. 
Let $I$ be the set of facilities. Then, a location of $I$ on a graph $G=(V, E)$ is a tuple $X$ of $|I|$ points on $G$ (which are not necessarily distinct). We denote by $G^{I}$ the family of all the locations of $I$ on $G$. Suppose that each vertex $v \in V$ has a weight $w_{v, i} \in \mathbb{R}$ for a facility $i \in I$, where $\mathbb{R}$ is the set of all real numbers; the weight $w_{v, i}$ represents the demand of $v$ to the service provided by $i \in I$. For each vertex $v \in V$ and a location $X \in G^{I}$, the cost $\operatorname{cost}(v, X)$ of $v$ to receive the service from $X$ is defined as follows:

$$
\operatorname{cost}(v, X):=\sum_{i \in I} w_{v, i} \cdot \operatorname{dist}\left(v, x_{i}\right),
$$

where $x_{i}$ denotes the point on $G$ at which the facility $i \in I$ is placed by $X$. In this paper, we study the following problem:

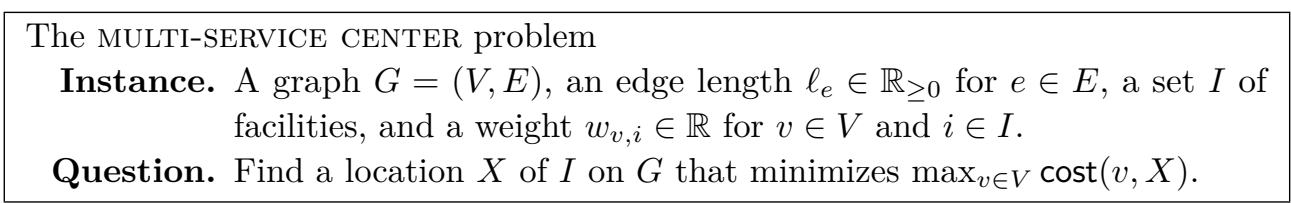

We call the problem $p$-SERVICE CENTER if the number $p$ of facilities is a fixed constant. In addition, we sometimes write the name of the problem with its restriction: For example, the problem is called MULTI-SERVICE CENTER with nonnegative weights if all weights $w_{v, i}$ are nonnegative for $v \in V$ and $i \in I$.

\section{Polynomial-Time Algorithm for Trees with Nonnegative Weights}

Recall that $\mathrm{Yu}$ and $\mathrm{Li}[10]$ showed that $p$-SERVICE CENTER with positive weights is solvable in polynomial time for general graphs, and 2-SERVICE CENTER with positive weights is solvable in $O(n \log n)$ time for trees having $n$ vertices. Both of the algorithms require that the number $p$ of facilities is fixed. In this section, we prove that MULTI-SERVICE CENTER with nonnegative weights is solvable in polynomial time for trees even when the number $p$ of facilities is taken as a part of input, as in the following theorem.

- Theorem 1. MULTI-SERVICE CENTER with nonnegative weights can be solved in polynomial time for trees.

In the remainder of this section, we prove Theorem 1. For notational convenience, we may assume that each edge of a given tree has a positive length; this assumption does not lose the generality because we simply regard each edge $e$ with $\ell_{e}=0$ as having a sufficiently small positive length.

\subsection{Technical highlights}

We first explain our main ideas and proof techniques briefly.

To describe a polynomial-time algorithm for trees, let us first consider the case when a graph is a path. In this case, it is not difficult to see that the problem can be reduced to a linear programming problem. In fact, we can identify a point on the path with a 1-dimensional coordinate $x$ by taking one of the end of the path as the origin. Then, the distance from each client to $x$ can be expressed by an absolute value function with respect to $x$. Therefore, MULTI-SERVICE CENTER for a path is equivalent to minimizing the maximum of the sum of absolute value functions with nonnegative coefficients, which can be formulated as a linear programming problem. 


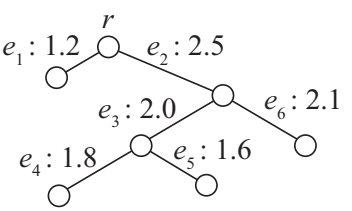

(a)

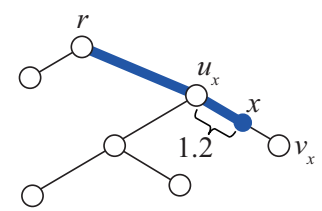

(b)

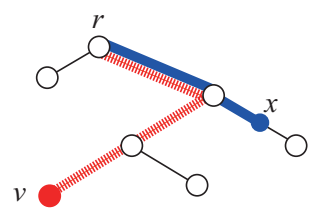

(c)

Figure 1 (a) A tree with edge-lengths, (b) the admissible vector $\tilde{x}=\left(\tilde{x}\left(e_{1}\right), \ldots, \tilde{x}\left(e_{6}\right)\right)^{\top}=$ $(0,2.5,0,0,0,1.2)^{\top}$ representing a point $x$, and (c) the admissible vector $\tilde{v}=(0,2.5,2.0,1.8,0,0)^{\top}$.

In order to extend the above observation to the tree case, we identify a point on a tree with a path from a specified vertex (a root). Then, we can represent a point on the tree by a vector in the $m$-dimensional space, where $m$ is the number of edges in the tree. (See Figure 1(b) as an intuitive example; a formal definition will be given later.) This representation gives us a linear programming problem to find $p$ vectors in the $m$-dimensional space, as formulated in Problem (3) later. However, since not all $m$-dimensional vectors correspond to a (feasible) point on the tree, the linear programming problem is a relaxation of MULTI-SERVICE CENTER. The key ingredient of our algorithm is to prove that the linear programming problem has in fact an optimal solution corresponding to an optimal facility location (Lemma 3). Since our proof is constructive, we can find an optimal facility location in polynomial time by solving the linear programming problem.

\subsection{Algorithm}

Let $T=(V, E)$ be a tree. We choose an arbitrary vertex $r$ in $V$ as the root of $T$, and regard $T$ as a rooted tree. For notational convenience, when we denote an edge $e$ by $e=u v$, we may assume that $u$ is the parent of $v$. For each vertex $v$ on $T$, we denote by $P_{v}$ the path in $T$ from $r$ to $v$. For each interior point $x$ of an edge $e_{x}=u_{x} v_{x}$, we denote by $P_{x}$ the path in $T$ from $r$ to $v_{x}$, that is, $P_{x}=P_{v_{x}}$. For each edge $e=u v$, let $T-e$ be the subgraph of $T$ obtained by deleting $e$ from $T$. Then, $T-e$ consists of exactly two trees that have $u$ and $v$, respectively; we denote the two trees by $T_{u}$ and $T_{v}$ where $u \in V\left(T_{u}\right)$ and $v \in V\left(T_{v}\right)$, respectively.

Let $x$ be any point on $T$, and assume that $x$ is located on an edge $e_{x}=u_{x} v_{x}$; note that $x=u_{x}$ or $x=v_{x}$ may hold. Then, we can express the point $x$ using a vector $\tilde{x}$ in $\mathbb{R}_{\geq 0}^{E}$, defined as follows (see Figure 1(b)):

$$
\tilde{x}(e)= \begin{cases}\ell_{e} & \text { if } e \in E\left(P_{u_{x}}\right)=E\left(P_{x}\right) \backslash\left\{e_{x}\right\}, \\ \operatorname{dist}\left(u_{x}, x\right) & \text { if } e=e_{x}, \\ 0 & \text { otherwise. }\end{cases}
$$

Conversely, we say that a vector $\tilde{x} \in \mathbb{R}_{\geq 0}^{E}$ is admissible if there exist an edge $e_{x}=u_{x} v_{x}$ and $d_{x}$ in $\left[0, \ell_{e_{x}}\right]$ such that $\tilde{x}$ has the form of (1) in which $\operatorname{dist}\left(u_{x}, x\right)$ is replaced with $d_{x}$. Then, there exists a one-to-one correspondence between a point $x \in T$ and an admissible vector $\tilde{x}$, and hence any point on $T$ can be represented as an admissible vector. When a vertex $v \in V$ and a point $x$ on $T$ are expressed by $\tilde{v} \in \mathbb{R}_{\geq 0}^{E}$ and $\tilde{x} \in \mathbb{R}_{\geq 0}^{E}$, respectively, it holds that

$$
\operatorname{dist}(v, x)=\sum_{e \in E}|\tilde{v}(e)-\tilde{x}(e)|
$$


(see also Figure 1(c)), because we have

$$
|\tilde{v}(e)-\tilde{x}(e)|= \begin{cases}\ell_{e} & \text { if } e \in\left(E\left(P_{x}\right) \triangle E\left(P_{v}\right)\right) \backslash\left\{e_{x}\right\} \\ \operatorname{dist}\left(u_{x}, x\right) & \text { if } e=e_{x} \notin E\left(P_{v}\right) \\ \operatorname{dist}\left(v_{x}, x\right) & \text { if } e=e_{x} \in E\left(P_{v}\right) \\ 0 & \text { otherwise. }\end{cases}
$$

For a vertex $v \in V$ and any vector $\tilde{x} \in \mathbb{R}_{\geq 0}^{E}$ (which is not necessarily admissible), we define

$$
d_{e}(v, \tilde{x})=|\tilde{v}(e)-\tilde{x}(e)|
$$

where $\tilde{v}$ is a vector expressing $v$ by (1). Consider the problem of finding $|I|$ vectors $\tilde{x}_{i} \in \mathbb{R}_{\geq 0}^{E}$ $(i \in I)$ that minimizes

$$
\max _{v \in V} \sum_{i \in I}\left(w_{v, i} \sum_{e \in E} d_{e}\left(v, \tilde{x}_{i}\right)\right)=\max _{v \in V} \sum_{i \in I}\left(w_{v, i} \sum_{e \in E}\left|\tilde{v}(e)-\tilde{x}_{i}(e)\right|\right)
$$

subject to $\tilde{x}_{i}(e) \in\left[0, \ell_{e}\right]$ for $i \in I$ and $e \in E$. Note that, by $(2)$, we have $\sum_{e \in E} d_{e}(v, \tilde{x})=$ $\operatorname{dist}(v, x)$ for any point $x$ on $T$ and its corresponding admissible vector $\tilde{x}$. Hence, if we have an additional constraint that each $\tilde{x}_{i}$ is admissible on the problem (3), then it is equivalent to MUlTi-SERVICE CENTER. Thus the problem (3) can be seen as a relaxation of MUlTI-SERVICE CENTER.

- Lemma 2. The optimal value of the problem (3) is smaller than or equal to that of MULTI-SERVICE CENTER with nonnegative weights.

Proof. Consider any optimal solution to MULTI-SERVICE CENTER with nonnegative weights which places each facility $i \in I$ at a point $x_{i}$ on $T$. Then, the corresponding vectors $\tilde{x}_{i}$ form a feasible solution of the problem (3), and its objective value is equal to the optimal value of MULTI-SERVICE CENTER with nonnegative weights because of (2). Thus, the statement holds.

We say that a feasible solution of the problem (3) is admissible if each vector $\tilde{x}_{i}(i \in I)$ of the solution is admissible. Then, an admissible solution of the problem (3) gives a location of $I$ on $T$. Lemma 2 and the following lemma ensure that solving the problem (3) is equivalent to solving MULTI-SERVICE CENTER with nonnegative weights.

- Lemma 3. The problem (3) has an admissible optimal solution. Furthermore, given an optimal solution $\tilde{x}_{i}(i \in I)$ to the problem (3), we can construct an admissible optimal solution in polynomial time.

Proof. Let $\tilde{x}_{i} \in \mathbb{R}_{>0}^{E}(i \in I)$ be an optimal solution to the problem (3). For each edge $e \in E$, let $P_{e}$ be the unique path in $T$ from the root $r$ to $e$ which does not include $e$ itself. Thus, $P_{e}=P_{u}$ for an edge $e=u v$. Let $F_{i}=\left\{e \in E \mid \tilde{x}_{i}(e)>0\right\}$. By definition, $\tilde{x}_{i}$ is admissible if and only if it satisfies the following conditions:

(A) any two distinct edges $e_{1}, e_{2} \in F_{i}$ satisfy either $e_{1} \in E\left(P_{e_{2}}\right)$ or $e_{2} \in E\left(P_{e_{1}}\right)$; and

(B) for each edge $e \in F_{i}$, there is no edge $e^{\prime} \in E\left(P_{e}\right)$ such that $\tilde{x}_{i}\left(e^{\prime}\right)<\ell_{e^{\prime}}$.

Suppose that $\tilde{x}_{i}$ is not admissible for some $i \in I$. We will show that we can modify the vector $\tilde{x}_{i}$ in polynomial time so that the resulting vector is admissible (i.e., satisfies both (A) and (B) above), without increasing the objective value of (3). 


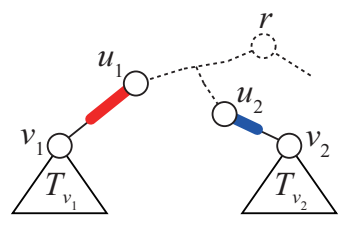

(a)

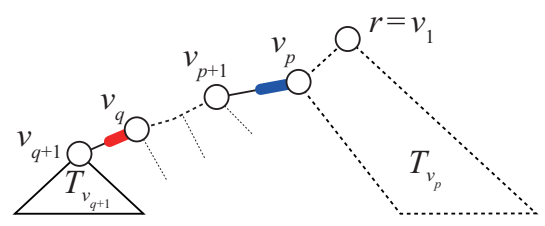

(b)

Figure 2 Illustration for the proof of Lemma 3.

We first modify $\tilde{x}_{i}$ so that it satisfies (A). Suppose that there exist two distinct edges $e_{1}=u_{1} v_{1}$ and $e_{2}=u_{2} v_{2}$ in $F_{i}$ such that both $e_{1} \notin E\left(P_{e_{2}}\right)$ and $e_{2} \notin E\left(P_{e_{1}}\right)$ hold. (See Figure 2(a).) Define a new vector $\tilde{x}_{i}^{\prime}$ by

$$
\tilde{x}_{i}^{\prime}(e)= \begin{cases}\tilde{x}_{i}(e)-\varepsilon & \text { if } e \in\left\{e_{1}, e_{2}\right\} \\ \tilde{x}_{i}(e) & \text { otherwise }\end{cases}
$$

where $\varepsilon=\min \left\{\tilde{x}_{i}\left(e_{1}\right), \tilde{x}_{i}\left(e_{2}\right)\right\}$. Then, $\tilde{x}_{i}^{\prime}(e) \in\left[0, \ell_{e}\right]$ for each $e \in E$, and hence $\tilde{x}_{i}^{\prime}$ is feasible to (3). We now claim that this modification does not increase the objective value as follows. For each vertex $z \in V$ expressed by $\tilde{z} \in \mathbb{R}_{\geq 0}^{E}$ and an index $q \in\{1,2\}$, we have

$$
\tilde{z}\left(e_{q}\right)= \begin{cases}0 & \text { if } z \in V\left(T_{u_{q}}\right) \\ \ell_{e_{q}} & \text { if } z \in V\left(T_{v_{q}}\right)\end{cases}
$$

where we recall that $T_{u_{q}}$ and $T_{v_{q}}$ are trees in $T-e_{q}$ such that $u_{q} \in V\left(T_{u_{q}}\right)$ and $v_{q} \in V\left(T_{v_{q}}\right)$. We thus have

$$
d_{e_{q}}\left(z, \tilde{x}_{i}^{\prime}\right)-d_{e_{q}}\left(z, \tilde{x}_{i}\right)= \begin{cases}-\varepsilon & \text { if } z \in V\left(T_{u_{q}}\right) \\ \varepsilon & \text { if } z \in V\left(T_{v_{q}}\right)\end{cases}
$$

Since $V\left(T_{v_{1}}\right) \cap V\left(T_{v_{2}}\right)=\emptyset$, the vertex $z$ is contained in $V\left(T_{u_{1}}\right)$ or $V\left(T_{u_{2}}\right)$. Therefore, it holds that

$$
\sum_{e \in E} d_{e}\left(z, \tilde{x}_{i}^{\prime}\right)-\sum_{e \in E} d_{e}\left(z, \tilde{x}_{i}\right)=d_{e_{1}}\left(z, \tilde{x}_{i}^{\prime}\right)-d_{e_{1}}\left(z, \tilde{x}_{i}\right)+d_{e_{2}}\left(z, \tilde{x}_{i}^{\prime}\right)-d_{e_{2}}\left(z, \tilde{x}_{i}\right) \leq 0 .
$$

In this way, while $F_{i}$ violates $(\mathrm{A})$, we can repeatedly replace $\tilde{x}_{i}$ with $\tilde{x}_{i}^{\prime}$ as above. Since this procedure decreases $\left|F_{i}\right|$ monotonically, the number of repetition is at most $\left|F_{i}\right| \leq|E|$. Thus we can obtain $\tilde{x}_{i}$ satisfying $(\mathrm{A})$ in polynomial time.

We next modify $\tilde{x}_{i}$ so that it also satisfies $(\mathrm{B})$. If $F_{i}=\emptyset$, then $\tilde{x}_{i}(e)=0$ for any $e \in E$, and hence $\tilde{x}_{i}$ is admissible. Otherwise, since $\tilde{x}_{i}$ satisfies $(\mathrm{A})$, all the edges in $F_{i}$ are on some path $P$ from $r$. Let $V(P)=\left\{v_{1}, v_{2}, \ldots, v_{k+1}\right\}$ and $E(P)=\left\{e_{1}, e_{2}, \ldots, e_{k}\right\}$ be the vertex set and the edge set of $P$, respectively, such that $v_{1}=r$ and $e_{j}=v_{j} v_{j+1}$ for $j=1, \ldots, k$. (See Figure 2(b).) Define $p:=\min \left\{j \in\{1, \ldots, k\} \mid \tilde{x}_{i}\left(e_{j}\right)<\ell_{e_{j}}\right\}$; let $p=+\infty$ if such $j$ does not exist. Define $q:=\max \left\{j \in\{1, \ldots, k\} \mid \tilde{x}_{i}\left(e_{j}\right)>0\right\}$; such $j$ always exists because $F_{i} \subseteq E(P)$. Note that $\tilde{x}_{i}$ satisfies (B) if and only if $p \geq q$. Suppose that $\tilde{x}_{i}$ does not satisfy (B), that is, $p<q$. Then, $p \neq+\infty$ holds, and hence we have $\tilde{x}_{i}\left(e_{p}\right)<\ell_{e_{p}}$. Define a new vector $\tilde{x}_{i}^{\prime}$ by

$$
\tilde{x}_{i}^{\prime}(e)= \begin{cases}\tilde{x}_{i}(e)+\varepsilon & \text { if } e=e_{p} \\ \tilde{x}_{i}(e)-\varepsilon & \text { if } e=e_{q} \\ \tilde{x}_{i}(e) & \text { otherwise }\end{cases}
$$


where $\varepsilon=\min \left\{\ell_{e_{p}}-\tilde{x}_{i}\left(e_{p}\right), \tilde{x}_{i}\left(e_{q}\right)\right\}$. Then, $\tilde{x}_{i}^{\prime}(e) \in\left[0, \ell_{e}\right]$ for each $e \in E$, and hence $\tilde{x}_{i}^{\prime}$ is feasible to (3). We now claim that this modification does not increase the objective value as follows. For any vertex $z \in V$, we have

$$
d_{e_{p}}\left(z, \tilde{x}_{i}^{\prime}\right)-d_{e_{p}}\left(z, \tilde{x}_{i}\right)= \begin{cases}\varepsilon & \text { if } z \in V\left(T_{v_{p}}\right) \\ -\varepsilon & \text { if } z \in V\left(T_{v_{p+1}}\right)\end{cases}
$$

and

$$
d_{e_{q}}\left(z, \tilde{x}_{i}^{\prime}\right)-d_{e_{q}}\left(z, \tilde{x}_{i}\right)= \begin{cases}-\varepsilon & \text { if } z \in V\left(T_{v_{q}}\right) \\ \varepsilon & \text { if } z \in V\left(T_{v_{q+1}}\right)\end{cases}
$$

where we recall that $T_{v_{p}}$ and $T_{v_{p+1}}$ are trees in $T-e_{p}$ such that $v_{p} \in V\left(T_{v_{p}}\right)$ and $v_{p+1} \in$ $V\left(T_{v_{p+1}}\right)$, and recall that $T_{v_{q}}$ and $T_{v_{q+1}}$ are trees in $T-e_{q}$ such that $v_{q} \in V\left(T_{v_{q}}\right)$ and $v_{q+1} \in V\left(T_{v_{q+1}}\right)$. Since $V\left(T_{v_{p}}\right) \cap V\left(T_{v_{q+1}}\right)=\emptyset$, we can see that the objective value does not increase similarly to (4).

Therefore, we can repeat replacing $\tilde{x}_{i}$ with $\tilde{x}_{i}^{\prime}$ as above while $\tilde{x}_{i}$ violates (B). Since this procedure either increases $p$ or decreases $q$ monotonically, we can finally obtain $\tilde{x}_{i}$ satisfying $p \geq q$, that satisfies (B), in polynomial time.

In this way, we can obtain an optimal solution $x$ that is admissible in polynomial time.

We are now ready to prove Theorem 1 .

Proof of Theorem 1. It follows from Lemmas 2 and 3 that it suffices to solve the problem (3). Note that $\sum_{i \in I}\left(w_{v, i} \sum_{e \in E}\left|\tilde{v}(e)-\tilde{x}_{i}(e)\right|\right)$ is a separable-convex function. Since the maximum of convex functions is also convex, so is the objective function of (3). Therefore, the problem (3) is a convex programming problem, which can be solved in polynomial time (see e.g., [3]).

In fact, we can reduce the problem (3) to the following linear programming problem:

minimize $\quad c$

$$
\begin{aligned}
\text { subject to } & \sum_{i \in I}\left(w_{v, i} \sum_{e \in E}\left|\tilde{v}(e)-\tilde{x}_{i}(e)\right|\right) \leq c \quad(v \in V), \\
& \tilde{x}_{i}(e) \leq \ell_{e} \quad(i \in I, e \in E), \\
& \tilde{x}_{i} \in \mathbb{R}_{\geq 0}^{E} \quad(i \in I), \\
& c \in \mathbb{R}_{\geq 0},
\end{aligned}
$$

where $\tilde{x}_{i}(e)(i \in I, e \in E)$ and $c$ are variables. Note that the first constraint can be described by linear inequalities, since the left-hand side is

$$
\sum_{i \in I}\left(w_{v, i} \sum_{e \in E}\left|\tilde{v}(e)-\tilde{x}_{i}(e)\right|\right)=\sum_{i \in I} w_{v, i}\left(\sum_{e \in E\left(P_{v}\right)}\left(\ell_{e}-\tilde{x}_{i}(e)\right)+\sum_{e \in E \backslash E\left(P_{v}\right)} \tilde{x}_{i}(e)\right) .
$$

Therefore, it is a linear programming problem with polynomial size, which can be solved in polynomial time (see e.g., [9]).

\section{$4 \quad$ Hardness Results}

In this section, we show that MULTI-SERVICE CENTER is computationally intractable even for very restricted instances. We emphasize again that our analyses are sharp in contrast to Theorem 1. 


\subsection{Technical Highlights}

Recall that, in MULTI-SERVICE CENTER, we are allowed to place each facility at any point on a graph (even on an edge), which makes a solution flexible. We design reductions so that reduced instances force all facilities to be placed at only vertices in any optimal solution. To ensure this condition, we need to analyze the structure of optimal solutions carefully. Interestingly, we will verify this condition for cycles (Theorem 7) by using the nonsingularity of a "distance matrix" [2], which has been studied in the area of algebraic graph theory.

\subsection{NP-hardness for paths and stars with negative weights}

In this subsection, we show that MULTI-SERVICE CENTER is intractable even for paths and stars if weights of vertices take negative integers. More specifically, the problem is NP-hard for paths of only three vertices, and is strongly NP-hard for stars. Indeed, a path of three vertices is a star, and hence we will construct a common reduction from the following problem:

The EQUALLY PARTITION problem

Instance. A set $A$ of elements, a bound $b \in \mathbb{Z}_{\geq 0}$, and a size $s_{i} \in \mathbb{Z}_{\geq 0}$ for each $i \in A$ such that $\sum_{i \in A} s_{i}=m b$ for some positive integer $m$.

Question. Can $A$ be partitioned into $m$ disjoint sets $A_{1}, A_{2}, \ldots, A_{m}$ such that $\sum_{i \in A_{j}} s_{i}=b$ for all $j \in\{1,2, \ldots, m\} ?$

Here, $\mathbb{Z}_{\geq 0}$ is the set of all nonnegative integers. We summarize our reduction from EQUALLY PARTITION to MULTI-SERVICE CENTER as in the following theorem.

- Theorem 4. There is a polynomial-time reduction from EQUALLY PARTITION to MULTISERVICE CENTER for instances such that

(a) $G=(V, E)$ is a star $K_{1, m}$ with the center vertex $r$ having $m$ leaves;

(b) $\ell_{e}:=1$ for every $e \in E$;

(c) $I:=A$; and

(d) for $v \in V$ and $i \in I(=A)$,

$$
w_{v, i}:= \begin{cases}-s_{i} & \text { if } v \in V \backslash\{r\} \\ -s_{i} \cdot \frac{2(m-1)}{m} & \text { if } v=r .\end{cases}
$$

Notice that EQUALLY PARTITION corresponds to an NP-hard problem PARTITION [5, SP12] if $m=2$. In addition, for general $m$, EQUALLY PARTITION contains all instances of a strongly NP-hard problem 3-PARTITION [5, SP15]. Thus, the following corollary can be obtained from Theorem 4.

- Corollary 5. The following (i) and (ii) hold.

(i) Multi-SERviCE CEnTER is NP-hard even when $G=(V, E)$ is a path of three vertices, $\ell_{e}=1$ for every $e \in E$, and $w_{v, i}=w_{v^{\prime}, i}$ for any $v, v^{\prime} \in V$ and $i \in I$.

(ii) Multi-Service Center is NP-hard in the strong sense even when $G=(V, E)$ is a star, and $\ell_{e}=1$ for every $e \in E$.

As described in Theorem 4, our reduction from EQUALLY PARTITION to MULTI-SERVICE CENTER is as follows. Suppose that we are given an instance of EQUALLY PARTITION, that is, a set $A$ of elements, a bound $b \in \mathbb{Z}_{\geq 0}$, and a size $s_{i} \in \mathbb{Z}_{\geq 0}$ for each $i \in A$ such that $\sum_{i \in A} s_{i}=m b$. Then, we construct a corresponding instance of MULTI-SERVICE CENTER 
as follows. Let $G=(V, E)$ be a star $K_{1, m}$ with the center vertex $r$ and having $m$ leaves $v_{1}, v_{2}, \ldots, v_{m}$. Set $\ell_{e}:=1$ for every $e \in E$, and define $I:=A$. For $v \in V$ and $i \in I(=A)$, set

$$
w_{v, i}:= \begin{cases}-s_{i} & \text { if } v \in V \backslash\{r\} ; \\ -s_{i} \cdot \frac{2(m-1)}{m} & \text { if } v=r .\end{cases}
$$

This reduction can be done in polynomial time.

To show the correctness of our reduction above, it suffices to prove the following lemma.

- Lemma 6. The original instance of EQUALLY PARTITION has a desired partition if and only if there is a location $X$ of I for the corresponding instance of MULTI-SERVICE CENTER such that $\max _{v \in V} \operatorname{cost}(v, X) \leq-2(m-1) b$.

Proof. Necessity ("only if" part). Suppose that the original instance of EQUALLY PARTITION has a partition $\left(A_{1}, A_{2}, \ldots, A_{m}\right)$ of $A$ such that $\sum_{i \in A_{j}} s_{i}=b$ for all $j \in\{1,2, \ldots, m\}$. In this case, we place the facilities in $A_{j}$ at the vertex $v_{j} \in V$, that is, for each $i \in A_{j}$, we define $x_{i}:=v_{j}$ in the corresponding instance of MULTi-SERvice Center. Since $\left(A_{1}, A_{2}, \ldots, A_{m}\right)$ is a partition of $A=I$, this properly defines a location $X$ of $I$. Then, for each leaf $v_{j} \in V \backslash\{r\}$, we can estimate the cost of $v$ to receive the service from $X$ as follows:

$$
\operatorname{cost}\left(v_{j}, X\right)=\sum_{i \in I} w_{v_{j}, i} \cdot \operatorname{dist}\left(v_{j}, x_{i}\right)=2 \cdot \sum_{i \in I \backslash A_{j}}\left(-s_{i}\right)=-2(m-1) b .
$$

Similarly, for the center vertex $r$ of the star, its cost can be estimated as follows:

$$
\operatorname{cost}(r, X)=\sum_{i \in I} w_{r, i} \cdot \operatorname{dist}\left(r, x_{i}\right)=\sum_{i \in I}\left(-s_{i} \cdot \frac{2(m-1)}{m}\right)=-2(m-1) b .
$$

Therefore, $X$ is a location of $I$ for the corresponding instance of MULTI-SERVICE CENTER such that $\max _{v \in V} \operatorname{cost}(v, X) \leq-2(m-1) b$, as required.

Sufficiency ("if" part). Suppose that there is a location $X \in G^{I}$ of $I$ for the corresponding instance of MULTI-SERVICE CENTER such that $\max _{v \in V} \operatorname{cost}(v, X) \leq-2(m-1) b$. For each facility $i \in I$, let $x_{i}$ denote the point on $G$ at which $i$ is placed by $X$. Since $r$ is the center vertex of the star, $\operatorname{dist}\left(r, x_{i}\right) \leq 1$ for any $i \in I$. In addition, since $w_{r, i}$ is negative for any $i \in I$, we have

$$
\operatorname{cost}(r, X)=\sum_{i \in I} w_{r, i} \cdot \operatorname{dist}\left(r, x_{i}\right) \geq \sum_{i \in I}\left(-s_{i} \cdot \frac{2(m-1)}{m}\right)=-2(m-1) b .
$$

Since we have assumed that $\max _{v \in V} \operatorname{cost}(v, X) \leq-2(m-1) b$, the inequality above is tight. We thus have $\operatorname{dist}\left(r, x_{i}\right)=1$ for any $i \in I$. Observe that $\operatorname{dist}\left(r, x_{i}\right)=1$ means that $x_{i}$ is equal to one of the points $v_{1}, v_{2}, \ldots, v_{m}$. With this observation, we obtain a partition $\left(A_{1}, A_{2}, \ldots, A_{m}\right)$ of $A$ by defining $A_{j}:=\left\{i \in I \mid x_{i}=v_{j}\right\}$ for each $j \in\{1,2, \ldots, m\}$.

We now claim that $\sum_{i \in A_{j}} s_{i}=b$ for all $j \in\{1,2, \ldots, m\}$, and hence $\left(A_{1}, A_{2}, \ldots, A_{m}\right)$ is a desired partition for EQUALLY PARTITION. To see this, we evaluate $\operatorname{cost}\left(v_{j}, X\right)$ as follows:

$$
\begin{aligned}
\max _{j \in\{1,2, \ldots, m\}} \operatorname{cost}\left(v_{j}, X\right) & =\max _{j \in\{1,2, \ldots, m\}}\left(\sum_{i \in I} w_{v_{j}, i} \cdot \operatorname{dist}\left(v_{j}, x_{i}\right)\right) \\
& \geq \frac{1}{m} \sum_{j=1}^{m} \sum_{i \in I} w_{v_{j}, i} \cdot \operatorname{dist}\left(v_{j}, x_{i}\right)=\frac{1}{m} \sum_{j=1}^{m} \sum_{i \in I \backslash A_{j}}\left(-s_{i}\right) \cdot 2 \\
& =-\frac{2}{m} \sum_{i \in I}(m-1) s_{i}=-2(m-1) b .
\end{aligned}
$$


Since we have assumed that $\max _{v \in V} \operatorname{cost}(v, X) \leq-2(m-1) b$, the inequality above is tight. Then, the tightness of the inequality shows that $\sum_{i \in I} w_{v_{j}, i} \operatorname{dist}\left(v_{j}, x_{i}\right)=-2(m-1) b$ for every $v_{j} \in V \backslash\{r\}$. Therefore, we have

$$
-2(m-1) b=\sum_{i \in I} w_{v_{j}, i} \cdot \operatorname{dist}\left(v_{j}, x_{i}\right)=\sum_{i \in I \backslash A_{j}}\left(-s_{i}\right) \cdot 2=-2\left(m b-\sum_{i \in A_{j}} s_{i}\right) .
$$

We thus have $\sum_{i \in A_{j}} s_{i}=b$ for all $j \in\{1,2, \ldots, m\}$, as claimed.

This completes the proof of Theorem 4, and hence Corollary 5 follows.

\subsection{Strong NP-hardness for cycles with nonnegative weights}

We show that the problem is strongly NP-hard even when restricted to cycles with identical edge-length and nonnegative integer weights.

- Theorem 7. MULTI-SERVICE CENTER with nonnegative weights is NP-hard in the strong sense even when $G=(V, E)$ is a cycle, $\ell_{e}=1$ for every $e \in E$, and $w_{v, i}=w_{v^{\prime}, i} \in \mathbb{Z}_{\geq 0}$ for any $v, v^{\prime} \in V$ and $i \in I$.

Thus, MULTI-SERVICE CENTER cannot be solved in pseudo-polynomial time even for such restricted instances unless $\mathrm{P}=\mathrm{NP}$.

In the remainder of this subsection, we prove the theorem by giving a polynomial-time reduction from a strongly NP-hard problem 3-PARTITION to MULTI-SERVICE CENTER for such restricted instances. The 3-PARTITION problem is defined as follows (see, e.g., [5, SP15]):

The 3-PARTITION problem

Instance. A set $A$ of $3 m$ elements, a bound $b \in \mathbb{Z}_{\geq 0}$, and a size $s_{i} \in \mathbb{Z}_{\geq 0}$ with $\frac{b}{4}<s_{i}<\frac{b}{2}$ for each $i \in A$ such that $\sum_{i \in A} s_{i}=m b$.

Question. Can $A$ be partitioned into $m$ disjoint sets $A_{1}, A_{2}, \ldots, A_{m}$ such that $\sum_{i \in A_{j}} s_{i}=b$ for all $j \in\{1,2, \ldots, m\}$ ?

Note that since $\frac{b}{4}<s_{i}<\frac{b}{2}$ for each $i \in A$, we have $\left|A_{j}\right|=3$ for all $j \in\{1,2, \ldots, m\}$. It is known that 3-PARTITION remains NP-hard in the strong sense even if $m$ is restricted to be odd [8].

Suppose that we are given a set $A$ of $3 m$ elements, a bound $b \in \mathbb{Z}_{\geq 0}$, and a size $s_{i} \in \mathbb{Z}_{\geq 0}$ for each $i \in A$ as an instance of 3-PARTition, where $m$ is an odd number. We construct a corresponding instance of MULTI-SERVICE CENTER as follows. Let $G=(V, E)$ be a cycle with $m$ vertices such that $V=\left\{v_{1}, v_{2}, \ldots, v_{m}\right\}, E=\left\{v_{1} v_{2}, v_{2} v_{3}, \ldots, v_{m-1} v_{m}, v_{m} v_{1}\right\}$, and $\ell_{e}:=1$ for every $e \in E$. Define $I:=A$, and set $w_{v, i}:=s_{i}$ for $v \in V$ and $i \in I(=A)$. This reduction can be done in polynomial time.

To show the correctness of our reduction above, it suffices to prove the following lemma.

- Lemma 8. The original instance of 3-PARTITION has a desired partition if and only if there is a location $X$ of I for the corresponding instance of MULTI-SERVICE CENTER such that

$$
\max _{v \in V} \operatorname{cost}(v, X) \leq \frac{\left(m^{2}-1\right) b}{4}
$$

Proof. Necessity ("only if" part). Suppose that the original instance of 3-PARTITION has a partition $\left(A_{1}, A_{2}, \ldots, A_{m}\right)$ of $A$ such that $\sum_{i \in A_{j}} s_{i}=b$ for all $j \in\{1,2, \ldots, m\}$. In this case, we place the (three) facilities in $A_{j}$ at the vertex $v_{j} \in V$, that is, for each $i \in A_{j}$, we define 
$x_{i}:=v_{j}$ in the corresponding instance of MULTI-SERviCE CEnTER. Since $\left(A_{1}, A_{2}, \ldots, A_{m}\right)$ is a partition of $A=I$, this properly defines a location $X$ of $I$. Then, for each vertex $v \in V$, we can estimate the cost of $v$ to receive the service from $X$ as follows:

$$
\begin{aligned}
\operatorname{cost}(v, X) & =\sum_{i \in I} w_{v, i} \cdot \operatorname{dist}\left(v, x_{i}\right)=\sum_{j=1}^{m}\left(\sum_{i \in A_{j}} w_{v, i} \cdot \operatorname{dist}\left(v, v_{j}\right)\right) \\
& =\sum_{j=1}^{m}\left(\operatorname{dist}\left(v, v_{j}\right) \sum_{i \in A_{j}} s_{i}\right)=b \sum_{j=1}^{m} \operatorname{dist}\left(v, v_{j}\right)=2 b \sum_{k=1}^{(m-1) / 2} k=\frac{\left(m^{2}-1\right) b}{4} .
\end{aligned}
$$

Therefore, $X$ is a location of $I$ for the corresponding instance of MULTI-SERVICE CENTER such that $\max _{v \in V} \operatorname{cost}(v, X) \leq \frac{\left(m^{2}-1\right) b}{4}$, as required.

Sufficiency ("if" part). Suppose that there is a location $X \in G^{I}$ of $I$ for the corresponding instance of MULTI-SERVICE CENTER such that $\max _{v \in V} \operatorname{cost}(v, X) \leq \frac{\left(m^{2}-1\right) b}{4}$. For each facility $i \in I$, let $x_{i}$ denote the point on $G$ at which $i$ is placed by $X$. We will prove the following (a) and (b):

(a) $X$ places all facilities in $I$ at vertices of $G$; and

(b) $\sum_{i: x_{i}=v_{j}} s_{i}=b$ for every $v_{j} \in V$.

Then, by defining $A_{j}:=\left\{i \in I \mid x_{i}=v_{j}\right\}$ for each $j \in\{1,2, \ldots, m\}$, we obtain a desired partition $\left(A_{1}, A_{2}, \ldots, A_{m}\right)$ of $A$ for the original instance of 3-PARTition.

We first prove (a). To see properties of the location $X$, we begin with the following claim.

- Claim 9. For any point $x$ on $G$, it holds that $\sum_{j=1}^{m} \operatorname{dist}\left(v_{j}, x\right) \geq \frac{m^{2}-1}{4}$. Furthermore, $\sum_{j=1}^{m} \operatorname{dist}\left(v_{j}, x\right)=\frac{m^{2}-1}{4}$ holds if and only if $x$ is a vertex of $G$.

Proof of the claim. Let $\varepsilon \geq 0$ be the distance from $x$ to the nearest vertex in $V$. Then,

$$
\sum_{j=1}^{m} \operatorname{dist}\left(v_{j}, x\right)=\sum_{k=1}^{(m-1) / 2}(k-\varepsilon)+\sum_{k=0}^{(m-1) / 2}(k+\varepsilon)=\frac{m^{2}-1}{4}+\varepsilon .
$$

This shows the claim, because $\varepsilon=0$ if and only if $x$ is a vertex of $G$.

By Claim 9, we have

$$
\begin{aligned}
\max _{v \in V} \operatorname{cost}(v, X) & =\max _{v \in V}\left(\sum_{i \in I} w_{v, i} \operatorname{dist}\left(v, x_{i}\right)\right) \\
& \geq \frac{1}{m} \sum_{v \in V} \sum_{i \in I} s_{i} \operatorname{dist}\left(v, x_{i}\right)=\frac{1}{m} \sum_{i \in I}\left(s_{i} \sum_{v \in V} \operatorname{dist}\left(v, x_{i}\right)\right) \\
& \geq \frac{1}{m} \sum_{i \in I}\left(s_{i} \cdot \frac{m^{2}-1}{4}\right)=\frac{\left(m^{2}-1\right) b}{4} .
\end{aligned}
$$

Since we have assumed that $\max _{v \in V} \operatorname{cost}(v, X) \leq \frac{\left(m^{2}-1\right) b}{4}$, all the inequalities above are tight. The tightness of the inequality in (5) shows that the point $x_{i}$ is a vertex of $G$ for each $i \in I$ by Claim 9 .

We then prove (b). Define $y_{u}:=\sum_{i: x_{i}=u} s_{i}$ for each $u \in V$, and define $y \in \mathbb{R}^{V}$ as the vector consisting of $y_{u}$ 's. The tightness of the above inequalities shows that $\sum_{i \in I} s_{i} \operatorname{dist}\left(v, x_{i}\right)=$ 
$\frac{\left(m^{2}-1\right) b}{4}$ for every $v \in V$, which is equivalent to

$$
\sum_{u \in V} y_{u} \operatorname{dist}(v, u)=\frac{\left(m^{2}-1\right) b}{4} \text { for every } v \in V \text {. }
$$

Let $D \in \mathbb{R}^{V \times V}$ be the distance matrix of $G$ defined by $D_{u v}=\operatorname{dist}(u, v)$ for $u, v \in V$. Then, (6) is represented as $D y=\frac{\left(m^{2}-1\right) b}{4} \cdot \mathbf{1}$, where $\mathbf{1}$ is the all-one vector in $\mathbb{R}^{V}$. Since $D \mathbf{1}=\frac{m^{2}-1}{4} \cdot \mathbf{1}$ by a simple calculation, we have

$$
D(y-b \mathbf{1})=\mathbf{0}
$$

It is shown in [2, Theorem 3.4] that the determinant of $D$ is equal to $\frac{m^{2}-1}{4}$, which implies that $D$ is nonsingular. Thus, (7) shows that $y=b \mathbf{1}$, that is, $y_{u}=b$ for every $u \in V$.

This completes the proof of Theorem 7 .

We finally note that our reductions indeed show that MULTI-SERVICE CENTER remains computationally hard even with an additional constraint that all facilities must be placed at only vertices.

\section{References}

1 Toshimitsu Anzai, Takehiro Ito, Akira Suzuki, and Xiao Zhou. The multi-service center decision problem is NP-complete for split graphs. In the 6th World Congress on Engineering and Technology (CET 2016), 2016.

2 Ravindra B. Bapat, Stephen J. Kirkland, and Michael Neumann. On distance matrices and Laplacians. Linear Algebra and Its Applications, 401:193-209, 2005.

3 Stephen Boyd and Lieven Vandenberghe. Convex Optimization. Cambridge University Press, New York, NY, USA, 2004.

4 Zvi Drezner and Horst W. Hamacher, editors. Facility Location: Applications and Theory. Springer-Verlag, Berlin Heidelberg, 2002.

5 Michael R. Garey and David S. Johnson. Computers and Intractability: A Guide to the Theory of NP-Completeness. W.H. Freeman \& Co., New York, NY, USA, 1990.

6 Teofilo F. Gonzalez. Clustering to minimize the maximum intercluster distance. Theoretical Computer Science, 38:293-306, 1985.

7 Dorit S. Hochbaum and David B. Shmoys. A best possible heuristic for the $k$-center problem. Mathematics of Operations Research, 10:180-184, 1985.

8 Sadish Sadasivam and Huaming Zhang. NP-completeness of st-orientations for plane graphs. Theoretical Computer Science, 411:995-1003, 2010.

9 Alexander Schrijver. Theory of Linear and Integer Programming. John Wiley \& Sons, Inc., New York, NY, USA, 1986.

10 Hung-I Yu and Cheng-Chung Li. The multi-service center problem. In the 23rd International Symposium on Algorithms and Computation (ISAAC 2012), volume 7676 of Lecture Notes in Computer Science, pages 578-587, 2012. 